\title{
WATER LEAKAGE DIAGNOSIS IN METRO TUNNELS BY INTERGRATION OF LASER POINT CLOUD AND INFRARED THERMAL IMAGING
}

\author{
Pengfei YU ${ }^{1}$, Hangbin WU ${ }^{1, *}$, Chun LIU ${ }^{1}$, Zhengwen XU ${ }^{2}$ \\ ${ }^{1}$ Colleage of Survey and Geo-Informatics Tongji University, Shanghai 200092, China; \\ ${ }^{2}$ Shanghai Geotechnical Investigations \& Design Institute Co .Ltd, Shanghai 200438, China
}

Commission III, WG III/5

KEY WORDS: Tunnel, Infrared Thermal Imaging, Laser Scanning, Intensity Data, Water Leakage Diagnosis

\begin{abstract}
:
Diagnosis of water leakage in metro tunnels is of great significance to the metro tunnel construction and the safety of metro operation. A method that integrates laser scanning and infrared thermal imaging is proposed for the diagnosis of water leakage. The diagnosis of water leakage in this paper is mainly divided into two parts: extraction of water leakage geometry information and extraction of water leakage attribute information. Firstly, the suspected water leakage is obtained by threshold segmentation based on the point cloud of tunnel. And the real water leakage is obtained by the auxiliary interpretation of infrared thermal images. Then, the characteristic of isotherm outline is expressed by solving Centroid Distance Function to determine the type of water leakage. Similarly, the location of leakage silt and the direction of crack are calculated by finding coordinates of feature points on Centroid Distance Function. Finally, a metro tunnel part in Shanghai was selected as the case area to make experiment and the result shown that the proposed method in this paper can be used to diagnosis water leakage disease completely and accurately.
\end{abstract}

\section{INTRODUCTION}

With metro operating time increases, diagnosis and prevention of tunnel disease has become an important operation to ensure the operational safety. Water leakage is one of the most common diseases in tunnels which not only affects the safety of circuit, but also causes pipe wall to fall off and destroys tunnel structure. Therefore, the timely and accurately diagnosis of water leakage in tunnels is of great significance.

Traditional method of diagnosis of water leakage in tunnels is visual inspection, but this method is inefficient, as well as influenced of human subjected. Therefore, diagnosis the water leakage in metro tunnels accurately and comprehensively has become a key issue in tunnel engineering.

Currently, diagnosis of water leakage by surveying means has gradually become main method. Quanhong Feng et al (Q. Feng, 2006) proposed that transformed the point cloud of tunnel into intensity images firstly and then extracted water leakage areas on the images of pipe wall according to the difference of laser intensity between water leakage and background area. However, the laser reflectivity of cable, structural support, paint and water proof coating in tunnel are similar to water. Therefore, this method may cause water leakage areas misjudgement. Based on digital images of water leakage, Xuezeng Liu et al (Xuezeng et al., 2012) used digital image processing technology such as grayscale transformation, threshold segmentation to extract water leakage and at the same time, through calibration experiments in laboratory, calibration parameters were calculated and the conversion from water leakage area in digital images to actual area was built. However, the type of water leakage and the leakage location can't be determined by this method. According to the physical characteristics of pipe wall after seepage and water's own special physical characteristics,
Shufei Cheng et al (Shufei and Hongwei, 2014) proposed two new methods of long-term automatic detection of water leakage in tunnel: temperature gradient method and conductivity method. However, these two methods have the defects of sensor deployment and inability to measure the area of water leakage automatically.

In addition to the above method, infrared thermography as a non-destructive detection technology has been applied to disease detection of infrastructure. Clark (Clark et al., 2003) used this technique to identify the water leakage on bridge successfully. Dou Haitao (Haitao et al., 2011) analysed water temperature, water flow rate and other influencing factors of infrared characteristics of water leakage through infrared thermal imaging technology. It can be seen that infrared thermal imaging technology can effectively identify water leakage and make an overall analysis of disease. However, due to the limitation of hardware, thermal infrared images often have the defects of low resolution and low signal to noise ratio which causing target on images cannot be measured.

Currently, water leakage can't be diagnosed accurately and completely by using single type sensor. To solve this problem, a water leakage diagnosis method which integrates laser scanning and thermal infrared imaging is proposed. Firstly, laser scanning is used to obtain the intensity information of point cloud of tunnel and extract the suspect water leakage areas. On this basis, by the auxiliary interpretation of the infrared image, real water leakage areas are extracted. At the same time, based on the temperature information provided by infrared thermal images, the temperature distribution characteristics of water leakage was analysed and the attribute information of water leakage such as water leakage type, location of leakage were extracted. Finally, experiment result showed that the proposed 
method in this paper can diagnose the water leakage disease in tunnels accurately and comprehensively.

\section{METHODOLOGY}

\subsection{Principle of water leakage diagnosis}

Laser beam emitted by scanner uses near-infrared band which resulting the laser intensity of water area is lower than the other targets. At the same time, point cloud collected by laser scanner not only contains coordinates information, but also has intensity information. Therefore, the difference of intensity between the water leakage and the surroundings in tunnels can be used to identify and separate the water leakage.

On the other hand, as the result of internal molecular heat movement of objects, temperature of all objects in nature are higher than absolute zero, and will be radiated energy outward. By Wein displacement law shows that the wavelength of object's maximum radiation is inversely proportional to its own temperature. When the wavelength corresponding to maximum radiance of object is the thermal infrared band, the temperature of object ranges from $-79.96{ }^{\circ} \mathrm{C}$ to $209.82^{\circ} \mathrm{C}$, covering the temperature of most objects under normal conditions. Therefore, according to Wein displacement law of displacement, infrared thermography can be used to obtain the temperature information of objects and their surroundings.

Water leakage in metro tunnels is mostly from ground so its own temperature is low. In addition, water has a large heat capacity, which results in a less susceptible temperature to the water and the evaporation of water entails the absorption of heat. The above reasons cause the difference between the temperature of water leakage and surroundings in tunnels providing the possibility for the water leakage diagnosis by infrared thermal imaging technology.

\subsection{Process of water leakage diagnosis}

The diagnosis of water leakage mainly divided into two parts in this paper: extraction of water leakage geometry information and extraction of water leakage attribute information, as Figure. 1 shows.

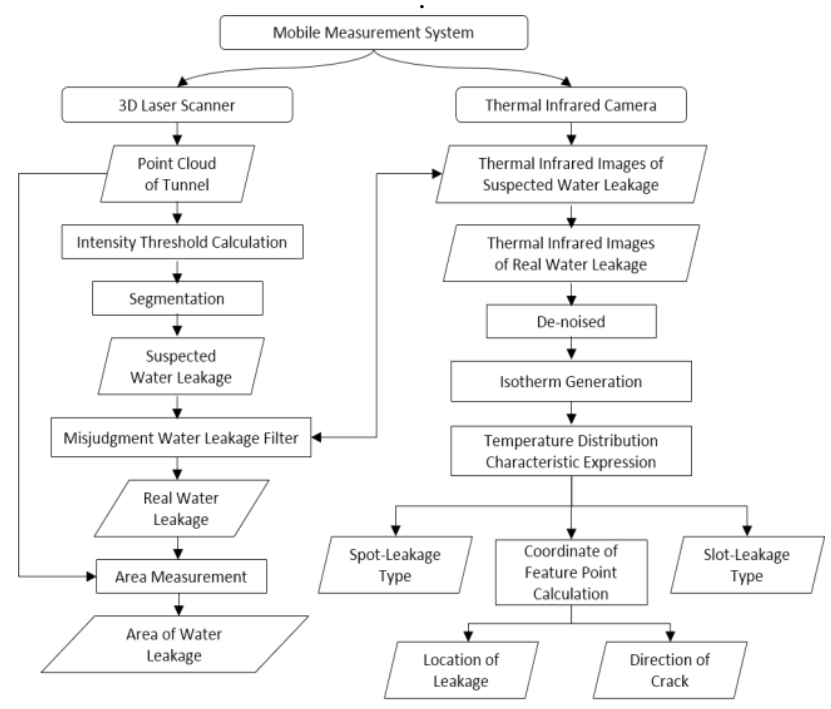

Figure 1. Flowchart of water leakage diagnosis
For extraction of water leakage geometry information, the method in this paper is as follows: firstly, intensity information of point cloud is taken to calculate intensity threshold value. Based on the threshold, segmentation method is used to extract the area of suspected water leakage. Then, infrared thermal images of the same region are obtained to filter out misjudgement area and get real water leakage. Finally, measuring area of real water leakage on intensity image of tunnel to get the accurate geometry information of water leakage.

As for extraction of water leakage attribute information, firstly, the thermal infrared images of water leakage are de-noised. Then based on images, isotherm of every water leakage is generated and the temperature distribution characteristics of water leakage is expressed by solving the Centroid Distance Function of isotherm outline. Finally, the location of leakage silt and the direction of cracks are calculated by finding coordinates of the feature points on Centroid Distance Function.

\subsection{Extraction method of water leakage geometry information}

The geometric information of water leakage in metro tunnel mainly refers to the area of water leakage on pipe wall. In order to extract accurate geometry information, it is necessary to identify the real water leakage and segment it accurately.

2.3.1 Suspected water leakage extraction: Based on the low intensity reflected by water leakage, Otsu (Nobuyuki Otsu, 1979) method is used to calculate the intensity threshold of laser points in the scanned area and then threshold segmentation is used for extracting water leakage. However, due to the laser reflectivity of cable, structural support, paint and waterproof coating in tunnels is similar to water, they will be mistaken divided into the water leakage. Therefore, based on intensity of point cloud, only suspected water leakage can be obtained.

2.3.2 Real water leakage extraction: When suspected water leakage is detected, thermal infrared images from same region are acquired by triggering an integrated thermal infrared camera on system. After scanning, for suspected water leakage, thermal infrared images of the same region are selected to help interpret. Then, based on the temperature characteristics of water leakage in thermal infrared images, misjudgement water leakage will be obtained. The method of extracting geometry information of water leakage is shown in Figure.2.

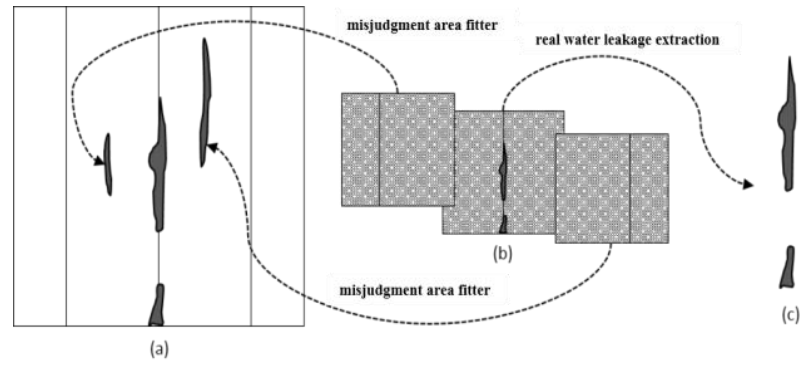

Figure 2. Extraction of water leakage geometry information. (a) Suspected water leakage extraction result. (b) Infrared thermal images of the same region. (c) Real water leakage extraction result

To facilitate the visualization and area measurement of water leakage in tunnel, Based on the method proposed in literature 
(Q.Feng, 2006), area of real water leakage are measured on the generated intensity image of tunnel.

\subsection{Extraction method of water leakage attribute information}

In order to diagnose water leakage comprehensive, in addition to the identification and extraction of water leakage, it's necessary to determine the leakage type, location of leakage and direction of leakage crack and other attribute information of disease attribute information.

2.4.1 Judgment of water leakage: This paper considers there are two types of water leakage commonly found in metro tunnel, namely spot-leakage type and slot-leakage type. Slotleakage can be divided into two subtypes, longitudinal-leakage type and transverse-leakage type. Due to different types of water leakage formation, different leakage location and expansion ways, its temperature distribution characteristics are quite different, as shown in Figure3.

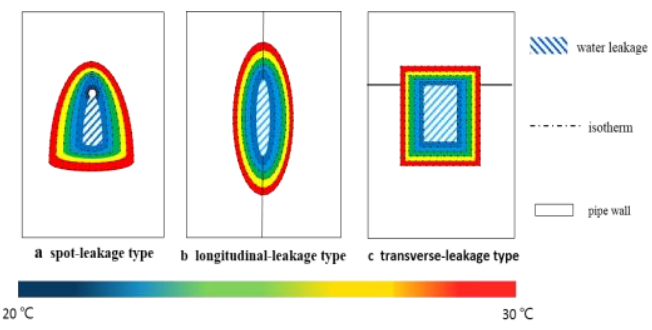

Figure 3. The temperature distribution characteristic of different type of water leakage

In order to express the temperature distribution of water leakage in thermal infrared images, firstly, the thermal infrared images of water leakage are de-noised. Then based on a fixed temperature interval value, temperature of each pixel is normalized to the corresponding temperature range to generate clear isothermal. The difference of temperature distribution characteristics can be expressed by different outline of isotherm. For example, lowest isotherm outline of spot-leakage type water leakage is approximately circular and its outer isotherm can be expressed as a quadratic curve. Isotherm outline of longitudinaltype and transverse-type water leakage can be approximated as ovals and rectangles, respectively. The morphological of outline information can be obtained by calculating the distance between the points on outer contour and its centroid called Centroid Distance Function. As shown in Figure.4, the curves are the Centroid Distance Function of circle's, oval's and rectangle's outline, respectively. The horizontal axis $\mathrm{k}$ represents each sampling point along the outline in a specific direction and vertical axis $d$ represents the distance.

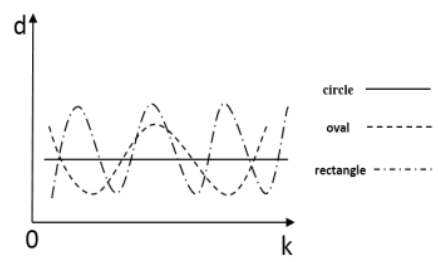

Figure 4. The Centroid Distance Function of different curves
2.4.2 Determination of leakage location and direction: According to 2.4.1, different type of water leakage has different Centroid Distance Function. The location of the leakage silt and the direction of crack can be obtained by getting coordinates of the feature points on isotherm outline. For the longitudinal-type water leakage, adjacent peak points of function represent the top and bottom vertices of the outline. The location of leakage and the direction of crack can be calculated by using the coordinates of these points. Similarly, the location and direction of transverse-type water leakage can be obtained by calculating the coordinates of adjacent peak points of the function.

\section{EXPERIMENT AND ANALYSIS}

\subsection{Protocols and equipment}

A metro tunnel part in Shanghai is selected as the case area. The length of experiment part is about $1.08 \mathrm{~km}$. To reduce the impact on metro operation, the experiment time was selected as January 18, 2017 0:00-3:00. In this experiment, Faro X330 laser scanner is used to collect point cloud of tunnel through mobile scanning. The average distance of point cloud of case tunnel is about $0.005 \mathrm{~m}$. HL640mini thermal infrared camera is used to collect the images of suspected water leakage areas. The temperature resolution of thermal camera is $0.05{ }^{\circ} \mathrm{C}$ and the temperature range is $-20^{\circ} \mathrm{C} \sim+150^{\circ} \mathrm{C}$. The spatial resolution of this camera is $0.68 \mathrm{rmad}$. In order to validate the method in this paper, water leakage areas are photographed by high-resolution visible light camera and confirmed by manual touch.

\subsection{Extraction of water leakage geometry information}

According to the introduction method of 2.3, the geometry information of water leakage is extracted. Based on the intensity of point cloud, intensity image of tunnel was generated as shown in Figure.5a. And Otsu method is used to segment the intensity image as shown in Figure.5b. Except cables and bottom parts of tunnel which are the non-water leakage can be artificial identified, eight suspected water leakage areas were extracted based on the intensity of point cloud. Through the interpretation of the infrared images at these eight areas, it is found that area7 and 8 didn't show obvious low temperature characteristics. Therefore, six real water leakage areas exists in this tunnel, as shown in Figure.5c and Figure.5d.

Visible light images of the suspected water leakage can confirm that area 1 to 6 are indeed water leakage. At the same time, the suspected water leakage 7,8 are actually waterproof coating after using artificial touch to determine. They are mistakenly divided into water leakage because they have the same laser reflection intensity with water. Therefore, the actual results are consistent with the extraction result of the method proposed in this paper. It is proved that the method is reliable for the extraction of water leakage geometry information. 
(a)

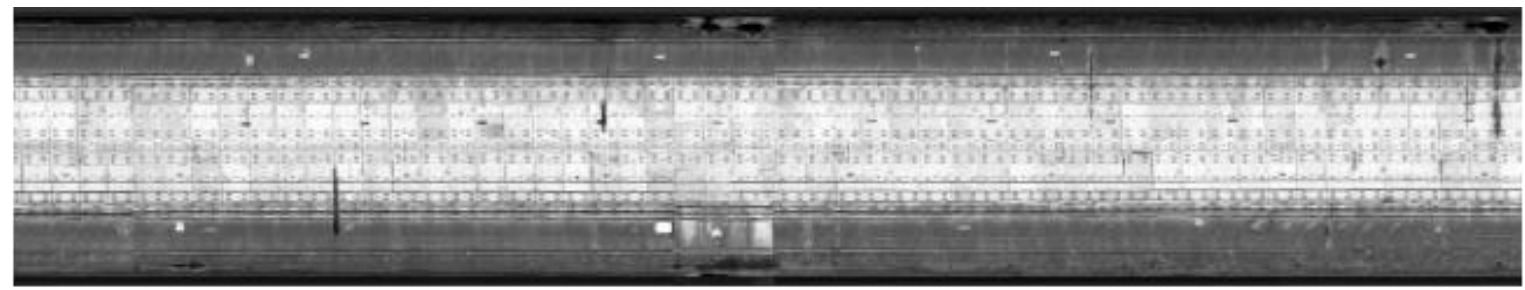

(b)

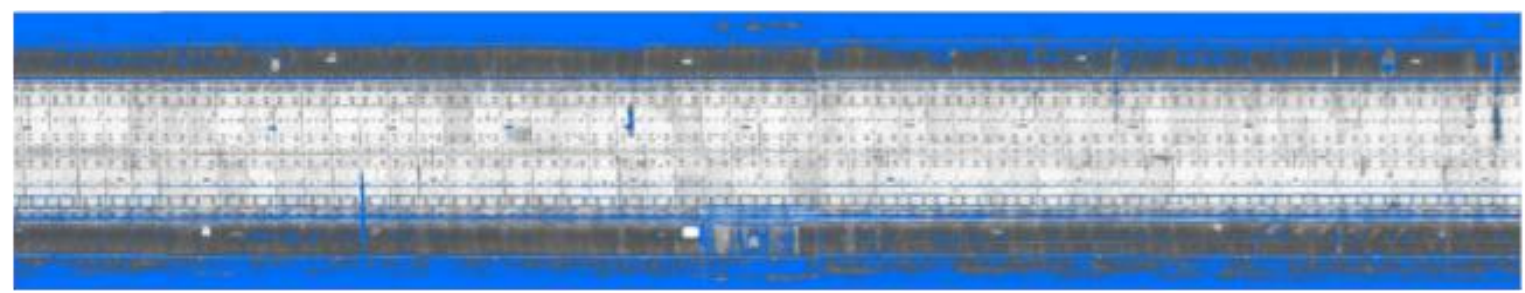

(c)
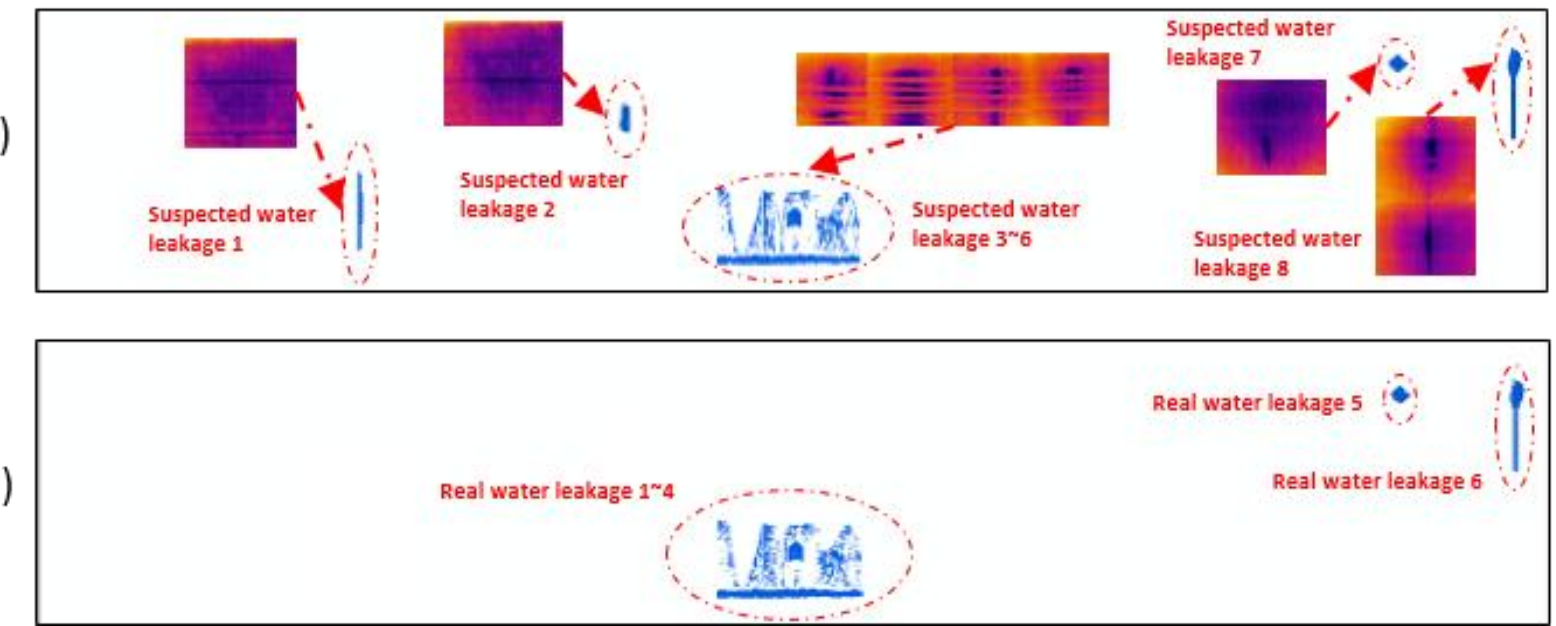

Figure 5. The extraction result of water leakage geometry information. (a) Intensity Image of Tunnel, (b) Threshold Segmentation Results Based on Point Cloud Intensity. (c) Extraction of Suspected Water Leakage \& Misjudgement Filter. (d) Extraction of Real Water Leakage

\subsection{Extraction of water leakage attribute information}

Two water leakage areas (real water leakage 1, 2 in Figure.5) are selected for attribute information extraction. Firstly thermal infrared images of two selected areas are de-noised and then the temperature of each pixel in thermal infrared images was normalized to the corresponding temperature rang at an interval of $0.5^{\circ} \mathrm{C}$ and the isotherm of them are generated.

The lowest isotherms of leakage 1 and 2 are selected to solve the Centroid Distance Function. According to the function information, it is determined that left part of leakage 1 is the longitudinal-leakage type water leakage and right part is the spot type water leakage. Leakage 2 is the transverse-leakage type water leakage. Through experiment, it has been found that outline of this type of water leakage's isotherm shows rectangular characteristics rarely because of the diffusion of temperature. Therefore, the outline of transverse-leakage type water leakage is more similar to the flat elliptical form. For the detected longitudinal and transverse cracks, based on coordinates of the feature points on corresponding functions, leakage silt location and the direction of crack are calculated and the extraction of water leakage attribute information was completed.
Finally, it was also confirmed through manual identification that transverse wall joint exist in the water leakage 2 and there are narrow longitudinal cracks on the left part of water leakage1 and three small leak spots on the right part. It can be seen that for this kind of mixed-type water leakage, accurate results can be obtained by using the method in this paper.

\subsection{Risk assessment of water leakage disease in case tunnel}

The type of water leakage was analysed and area of water leakage was count for all the six detected water leakage. The results are shown in Tab.1. Combining the requirements of the service status of structural sections in Code for Structural Appraisal of Shield Tunnel (Shanghai Urban and Rural Construction and Transportation Committee, 2013.): there exist few water leakage in this section and the amount of water leakage which area is smaller than $100 \mathrm{~m}^{2}$ doesn't exceed 7 , so the water-proof rating in this region is rated as 3rd. Therefore, according to the specification (Ministry of Housing and UrbanRural Development of the People's Republic of China, 2008), the service performance level of the tunnel in this interval is iii, indicating that the tunnel in this interval is already in the deterioration level. Although there is no security risk, it is urgent to manage. 
The International Archives of the Photogrammetry, Remote Sensing and Spatial Information Sciences, Volume XLII-3, 2018 ISPRS TC III Mid-term Symposium "Developments, Technologies and Applications in Remote Sensing", 7-10 May, Beijing, China

\begin{tabular}{c|c|c}
\hline Index & Type & $\begin{array}{c}\text { Area of } \\
\text { leakage }(\mathrm{m} 2)\end{array}$ \\
\hline 1 & longitudinal-leakage type & 0.75 \\
\hline 2 & longitudinal-leakage type \& spot-leakage type & 0.43 \\
\hline 3 & transverse- leakage type & 0.38 \\
\hline 4 & transverse- leakage type & 0.39 \\
\hline 5 & longitudinal- leakage type & 0.001 \\
\hline 6 & longitudinal-leakage type & 1.11 \\
\hline 7 & suspected type & -- \\
\hline 8 & suspected type & -- \\
\hline
\end{tabular}

Tab.1 The statistics of the type of seepage and the area of seepage in experiment tunnel

\section{CONCLUSION AND OUTLOOK}

In this paper, a method for water leakage diagnosis in metro tunnel using integrated laser scanning and infrared thermal imaging is proposed. Real water leakage is extracted by threshold segmentation of intensity image of tunnel and interpreting of thermal infrared images. The type of water leakage can be determined by analysing the Centroid Distance Function of its' isotherm. Similarly, the location of the leakage silt and the direction of crack are calculated by finding the coordinates of the feature points on the Centroid Distance Function. Experiments were carried out in a case tunnel in Shanghai. The results shown that the method proposed method in this paper can be used to diagnose the water leakage completely and accurately.

There are also some deficiencies in this paper, the determination of water leakage content is important for water leakage diagnosis. This article doesn't involve the study of it. Therefore, how to use laser scanning technology and thermal infrared technology to determine the water leakage content is the next search goal.

\section{ACKNOWLEDGEMENTS}

This project is funded by National Science Foundation of China (No. 41671451) National Science and Technology Major Program (2016YFB0502104)

\section{REFERENCES}

Clark, M.R., McCann, D.M. and Forde, M.C., 2003. Application of infrared thermography to the non-destructive testing of concrete and masonry bridges. NDT \& $E$ International, 36(4), pp. 265-275.

Haitao, D., Hongwei, H. and Yadong, X., 2011. Experiment Study of Factors Affecting Thermal Infrared Radiation Charateristics of Tunnel Lining Water Leakage. Chinese Journal of Rock Mechanics and Engineering (12), pp. 24262434.

Ministry of Housing and Urban-Rural Development of the People's Republic of China, 2008. Technical Code for Waterproofing of Underground Works: GB50108-2001

Nobuyuki Otsu., 1979. A threshold selection method from graylevel histograms. IEEE Trans. Sys, Man, Cyber, 9(1), pp. 62-66.

Q. Feng, G.W.K.R., 2006. Detection of water leakage using laser images from 3D laser scanning data, Proceedings of IAEG 10th Congress. London: The Geological Society of London, London.
Shanghai Urban and Rural Construction and Transportation Committee, 2013. Code for Structural Appraisal of Shield Tunnel: DG/TJ08-2123-2013

Shufei, C. and Hongwei, H., 2014. Monitoring Methods of Long-term Water Seepage in Shield Tunnel. Chinese Journal of Underground Space and Engineering, 10(3), pp. 733-738.

Xuezeng, L., Yunlong, S. and Yunfan, S., 2012. Detection Technology of Tunnel Leakage Disaster Based on Digital Image Processing. Chinese Journal of Underground Space and Engineering (S2), pp. 3779-3786. 